\section{Ocorrência de dor lombar e fatores associados em crianças e adolescentes de uma escola privada do sul do Brasil}

\author{
Low back pain and associated factors in children \\ and adolescents in a private school in \\ Southern Brazil
}

\author{
La aparición de dolor de espalda y los factores \\ asociados en niños y adolescentes en una \\ escuela privada en el sur de Brasil
}

\author{
1 Programa de Pós-graduação \\ em Ciências do Movimento \\ Humano, Universidade \\ Federal do Rio Grande do Sul. \\ Porto Alegre, Brasil. \\ Correspondência \\ A. T. Lemos \\ Laboratório de Pesquisa \\ do Exercício, Programa de \\ Pós-graduação em Ciências \\ do Movimento Humano, \\ Universidade Federal do Rio \\ Grande do Sul. \\ Rua Felizardo 750, sala 201, \\ Porto Alegre, RS 90690-200, \\ Brasil. \\ adrixlemos@gmail.com
}

\begin{abstract}
Studies have shown that children and adolescents with low back pain are also similarly affected when they reach adulthood, thus highlighting the importance of investigating causes of low back pain in school-age children. The study examined low back pain and associated factors in 770 schoolchildren 7 to 17 years of age in a private school in Porto Alegre, Rio Grande do Su State, Brazil. Low back pain was defined as pain or discomfort in the lumbar region in the previous month, assessed by a questionnaire. Low back pain was found in $31.6 \%$ of the subjects and was more prevalent in girls (41.9\%) than boys (21.4\%). Factors associated with lumbar pain were female gender, age 9 to 17 years, hyperactivity (borderline and abnormal categories), and emotional symptoms (abnormal category). Mapping the occurrence of low back pain and associated factors is important for identifying children and adolescents at risk and for developing effective programs for primary prevention.
\end{abstract}

Back Pain; Child; Adolescent; Risk Factors

\author{
Adriana Torres de Lemos 1 \\ Fábio Rosa dos Santos 1 \\ Rodrigo Baptista Moreira 1 \\ Débora Teixeira Machado \\ Fernando Cesar Camargo Braga 1 \\ Adroaldo Cezar Araujo Gaya ${ }^{1}$
}

\section{Resumo}

Estudos têm demonstrado que indivíduos que apresentam dor lombar na infância e adolescência são acometidos também na vida adulta, reforçando a importância de que as causas sejam investigadas desde a idade escolar. Avaliou-se a ocorrência de dor lombar e fatores associados em 770 escolares de 7 a 17 anos de idade de uma escola privada de Porto Alegre, Rio Grande do Sul, Brasil. A dor lombar foi definida como dor ou desconforto na região lombar no último mês e avaliada por meio de questionário. A ocorrência de dor lombar foi de 31,6\%, sendo mais prevalente no sexo feminino (41,9\%) do que no masculino (21,4\%). Os fatores associados à dor lombar foram sexo feminino, idade de 9 a 17 anos e os aspectos psicossociais hiperatividade (categorias limítrofe e anormal) e sintomas emocionais (categoria anormal). O mapeamento da ocorrência de dor lombar, bem como a investigação de seus fatores associados é de fundamental importância para a identificação de crianças e adolescentes em risco e para o desenvolvimento de programas eficazes de prevenção primária.

Dor nas Costas; Criança; Adolescente; Fatores de Risco 


\section{Introdução}

A dor lombar é definida como dor ou desconforto, localizada abaixo do arco costal e acima das pregas glúteas, sendo caracterizada como inespecífica quando não pode ser atribuída a causas definidas 1 . De acordo com o guia europeu para o manejo da dor lombar crônica inespecífica 2 , o percentual de indivíduos que em algum momento de suas vidas tiveram dor lombar é de até $84 \%$, sendo que após um episódio inicial, de $44 \%$ a $78 \%$ sofrem recidivas e de $26 \%$ a $37 \%$ se ausentam do trabalho. Ainda: causas conhecidas de dor lombar são responsáveis por menos de $15 \%$ das dores nas costas.

Walker ${ }^{3}$ reportou que na década passada os aumentos mais significativos na ocorrência de dor lombar seriam nos países em desenvolvimento, mas que a literatura carecia de informações sobre a prevalência nesses contextos. Verificar essa prevalência e a etiologia da dor lombar em países em desenvolvimento pode auxiliar no entendimento da sua causa global e em sua administração, podendo determinar se os fatores diferem dentre as características socioculturais ${ }^{4}$. Worku ${ }^{5}$ demonstrou que a maioria das publicações acerca desse tema tem sido realizada em países desenvolvidos, nos quais existe pouca heterogeneidade racial.

A prevalência de dor lombar em escolares se aproxima à referida em adultos 6 . Estudos têm demonstrado que indivíduos que apresentam dor lombar na infância e adolescência são acometidos também na vida adulta, o que originou maior interesse em investigar suas causas já nas idades iniciais 7,8. Sendo a dor lombar comum na adolescência e, sabendo-se que um percentual considerável (em torno de 25\%) apresenta sintomas persistentes, a capacidade de identificar as crianças e adolescentes que estão em risco fornece uma base de reflexão para intervenções preventivas 9 .

No cenário internacional, a ocorrência de dor lombar vem sendo amplamente investigada. Diversos estudos epidemiológicos apontam a prevalência de dor lombar, bem como possíveis fatores associados 7,10,11,12,13,14,15,16,17,18,19,20,21,22. Entretanto, não há consenso sobre as variáveis associadas, nem quanto aos meios de prevenção. Alguns dos fatores que vem sendo investigados são: sexo, idade, flexibilidade, força abdominal e de paravertebrais, atividades de vida diária, tabagismo, aspectos psicológicos, uso e peso de mochilas, hábitos de sono, prática de exercício físico, entre outros.

No Brasil, estudos com escolares já vêm demonstrando ocorrências preocupantes de dor lombar 23,24, porém eles ainda são escassos.
Assim, o objetivo deste estudo é descrever a ocorrência de dor lombar em escolares de 7 a 17 anos de idade, além de verificar sua associação com sexo, idade, prática de exercício e aspectos psicossociais.

\section{Materiais e métodos}

Este estudo transversal foi realizado com escolares de 7 a 17 anos de idade, matriculados em uma escola privada da cidade de Porto Alegre, Rio Grande do Sul, Brasil. Todos os estudantes da faixa etária em estudo foram convidados a participar, sendo a amostra constituída por 770 escolares. As avaliações foram realizadas durante as aulas de Educação Física por uma equipe previamente treinada. O projeto do estudo foi aprovado pelo Comitê de Ética da Universidade Federal do Rio Grande do Sul. Os pais ou responsáveis assinaram um Termo de Consentimento Livre e Esclarecido e os escolares participaram de forma voluntária.

Para a avaliação da dor lombar, utilizou-se questionário adaptado de Vidal 25 , cujas questões determinantes são semelhantes às utilizadas nos estudos de Jones et al. 26 e Watson et al. 20. Considerou-se que a dor lombar estava presente quando o escolar respondeu afirmativamente à questão "Durante o mês passado, você sentiu dor lombar (região pintada na figura abaixo) que tivesse duração de um dia ou mais?". Os resultados foram categorizados em dor lombar presente (resposta afirmativa à questão) e dor lombar ausente (resposta negativa à questão). A resposta à questão "Durante o mês passado, você sentiu dor nas costas que tivesse a duração de um dia ou mais?" foi observada com o propósito de confirmar a ocorrência de dor lombar. Os demais itens do questionário também foram considerados, tendo em vista que suas análises permitem verificar se a dor lombar não era decorrente do período menstrual, de traumas ou de patologias diagnosticadas. Logo, os escolares que responderam às questões de forma contraditória, que referiram traumatismos sem recuperação completa ou meninas que relacionaram a sua dor lombar ao período menstrual, foram excluídos da análise, o que totalizou 17 indivíduos (2,1\%). A objetividade e a fidedignidade do questionário foram testadas pelo índice de concordância da estatística kappa, em um subgrupo de 110 escolares selecionados aleatoriamente em cada sexo e idade, com intervalo de uma semana. Verificou-se excelente índice de concordância $(k>0,8)$ para cada uma das duas questões determinantes da dor lombar. A intensidade da dor lombar foi avaliada pela Escala 
de Faces, utilizada em crianças e adolescentes, cujos resultados poderiam variar de 0 (sem dor) a 5 (dor máxima). Assinalaram a escala apenas aqueles que haviam, na questão anterior, referido dor lombar. Também se questionou sobre a prática de exercício físico além das aulas de Educação Física, bem como a modalidade, local onde era praticada, frequência semanal e quantidade de horas por sessão. Caracterizou-se que o escolar realizava exercício físico além das aulas de Educação Física quando foi identificado que a prática era sistematizada e organizada. Entendeu-se, no presente estudo, como organizadas e sistematizadas as atividades que eram praticadas em academias, clubes ou escolinhas esportivas, por, no mínimo, uma hora por sessão e duas vezes por semana, sob orientação profissional. O outro grupo foi classificado como não praticante de exercício além das aulas de Educação Física, que ocorrem duas vezes por semana, por períodos de 50 minutos, nas quais são desenvolvidos elementos da aptidão física relacionada à saúde e ao desempenho motor, bem como a prática esportiva.

Para avaliação dos aspectos psicossociais, utilizou-se o questionário de capacidades e dificuldades - The Strengths and Difficulties Questionnaire (SDQ) 27. O SQD é uma ferramenta breve, de triagem comportamental, que propõe a compreensão dos comportamentos, emoções e relacionamentos de crianças e adolescentes. Ele inclui uma pontuação total (denominado neste estudo de aspectos psicossociais) e cinco dimensões (cada uma com pontuação de 0 a 10): quatro relacionadas ao comportamento "negativo" ou dificuldades (hiperatividade, sintomas emocionais, problemas de conduta e problemas de relacionamento com colegas) e uma com enfoque no comportamento "positivo" ou capacidades (comportamento pró-social). Utilizou-se a versão de autoavaliação para escolares de 10 a 17 anos e, para os escolares de 7 a 9 anos, a versão para pais/professores (tendo sido preenchido pelos professores), conforme recomendado. Os escores foram classificados em normal, limítrofe e anormal. A versão utilizada foi traduzida e validada para o português e encontra-se disponível no site do SQD 28.

Para o tratamento dos dados, utilizou-se frequência de ocorrência em valores absolutos e percentuais para as análises descritivas, estratificados por sexo. Para a identificação das variáveis associadas ao desfecho dor lombar e, a fim de serem estimadas suas razões de prevalência e intervalos de confiança, utilizou-se a regressão de Poisson, com variância robusta. Para a modelagem estatística, recorreu-se a modelos univariados de regressão de Poisson para analisar a relação isolada de cada variável com a dependente. Isso feito, foram selecionadas para a próxima etapa aquelas variáveis que apresentaram $o$ valor de $\mathrm{p}<0,25$, e o modelo final foi composto pelas variáveis que, no modelo multivariado, apresentaram significância (valor de p) menor que 0,05 . As variáveis independentes foram: sexo, idade, prática de exercício físico e aspectos psicossociais.

\section{Resultados}

Dos 770 escolares participantes do estudo, 50,4\% $(\mathrm{n}=388)$ eram do sexo masculino. O perfil antropométrico da amostra, estratificado por sexo e idade, está apresentado na Tabela 1. A ocorrência de dor lombar na amostra estudada foi de $31,6 \%$. No sexo masculino, $21,4 \%(n=83)$ referiram dor lombar e, no feminino, 41,9\% $(\mathrm{n}=160)$. A Figura 1 apresenta a frequência de dor lombar ao longo das idades.

Em relação à intensidade da dor, dentre os escolares $(n=243)$ que a referiram, 39,9\% e 29,2\% apontaram o nível dois e o três, respectivamente. Constatou-se ainda que aproximadamente $15 \%$ dos escolares referiram as intensidades quatro e cinco (Tabela 2). O zero na escala de faces significava a ausência de dor, tendo sido retirado da apresentação na Tabela 2 por ela demonstrar os valores percentuais apenas em relação aos escolares com dor e não em relação a todo grupo.

Os resultados da associação de cada variável ao desfecho (Tabela 3) demonstraram que o sexo, a idade, e os aspectos psicossociais hiperatividade, problemas de relacionamento com colegas, sintomas emocionais e problemas de conduta apresentaram valor de $\mathrm{p}$ menor que 0,25 , sendo selecionados para a etapa multivariada. O modelo final, composto por aquelas variáveis que na etapa multivariada apresentaram $\mathrm{p}<0,05$, está demonstrado na Tabela 4.

\section{Discussão}

A ocorrência de dor lombar encontrada no presente estudo foi de $31,6 \%$, sendo mais comum entre meninas $(41,9 \%)$ do que entre meninos $(21,4 \%)$. Dois estudos recentes com escolares brasileiros, de 11 a 14 anos 24 e de 15 a 18 anos 23 , encontraram ocorrências de 19,5\% e 49,3\%, respectivamente. O primeiro considera a dor lombar nos últimos 12 meses e o último não define dor lombar, apresentando as frequências para "todos os dias", semanal, mensal e "raramente". Diferenças na definição da dor lombar geram dificuldades na comparação entre prevalências 11 , 
Tabela 1

Perfil antropométrico da amostra, estratificado por sexo e idade.

\begin{tabular}{|c|c|c|c|c|c|c|c|c|}
\hline \multirow{2}{*}{$\begin{array}{l}\text { Idade } \\
\text { (anos) }\end{array}$} & \multicolumn{4}{|c|}{ Sexo masculino } & \multicolumn{4}{|c|}{ Sexo feminino } \\
\hline & $\mathrm{n}$ & $\begin{array}{l}\text { Estatura }(\mathrm{cm}) \\
\text { média } \pm \text { DP }\end{array}$ & $\begin{array}{l}\text { Massa }(\mathrm{kg}) \\
\text { média } \pm \text { DP }\end{array}$ & $\begin{array}{l}\mathrm{IMC}\left(\mathrm{kg} / \mathrm{m}^{2}\right) \\
\text { média } \pm \mathrm{DP}\end{array}$ & $\mathbf{n}$ & $\begin{array}{l}\text { Estatura }(\mathrm{cm}) \\
\text { média } \pm \text { DP }\end{array}$ & $\begin{array}{l}\text { Massa }(\mathrm{kg}) \\
\text { média } \pm \mathrm{DP}\end{array}$ & $\begin{array}{l}\mathrm{IMC}\left(\mathrm{kg} / \mathrm{m}^{2}\right) \\
\text { média } \pm \mathrm{DP}\end{array}$ \\
\hline 7 & 18 & $128,5 \pm 0,04$ & $27,3 \pm 4,75$ & $16,5 \pm 1,90$ & 18 & $125,5 \pm 0,08$ & $27,1 \pm 5,83$ & $17,1 \pm 2,25$ \\
\hline 8 & 24 & $133,4 \pm 0,06$ & $32,3 \pm 6,14$ & $18,0 \pm 2,50$ & 28 & $129,2 \pm 0,07$ & $29,7 \pm 6,34$ & $17,7 \pm 2,82$ \\
\hline 9 & 15 & $140,3 \pm 0,05$ & $37,2 \pm 8,53$ & $18,7 \pm 3,60$ & 29 & $1,372 \pm 0,07$ & $36,2 \pm 9,03$ & $19,1 \pm 3,95$ \\
\hline 10 & 38 & $142,7 \pm 0,06$ & $38,2 \pm 8,51$ & $18,6 \pm 3,01$ & 42 & $145,6 \pm 0,06$ & $40,2 \pm 7,92$ & $18,9 \pm 3,05$ \\
\hline 11 & 39 & $151,0 \pm 0,06$ & $46,4 \pm 10,9$ & $20,1 \pm 3,78$ & 33 & $153,1 \pm 0,07$ & $47,5 \pm 12,4$ & $20,2 \pm 3,87$ \\
\hline 12 & 44 & $156,4 \pm 0,10$ & $52,2 \pm 14,0$ & $21,2 \pm 5,61$ & 34 & $157,5 \pm 0,05$ & $50,6 \pm 9,36$ & $20,3 \pm 3,16$ \\
\hline 13 & 44 & $164,1 \pm 0,09$ & $59,2 \pm 16,5$ & $21,8 \pm 5,08$ & 45 & $159,8 \pm 0,05$ & $52,2 \pm 11,5$ & $20,4 \pm 4,14$ \\
\hline 14 & 47 & $169,6 \pm 0,06$ & $62,2 \pm 12,6$ & $21,5 \pm 3,87$ & 56 & $161,1 \pm 0,07$ & $53,8 \pm 8,55$ & $20,7 \pm 2,67$ \\
\hline 15 & 50 & $172,9 \pm 0,07$ & $66,6 \pm 12,9$ & $22,2 \pm 3,50$ & 49 & $160,9 \pm 0,07$ & $57,6 \pm 11,4$ & $22,1 \pm 3,69$ \\
\hline 16 & 53 & $173,2 \pm 0,06$ & $66,7 \pm 11,6$ & $22,2 \pm 3,80$ & 29 & $162,0 \pm 0,06$ & $58,9 \pm 9,77$ & $22,5 \pm 3,76$ \\
\hline 17 & 16 & $172,2 \pm 0,04$ & $65,6 \pm 6,00$ & $22,2 \pm 2,80$ & 19 & $160,7 \pm 0,06$ & $56,3 \pm 8,55$ & $21,8 \pm 2,98$ \\
\hline
\end{tabular}

DP: desvio-padrão; IMC: índice de massa corporal.

Figura 1

Percentual de ocorrência de dor lombar ao longo das idades, estratificada por sexo.

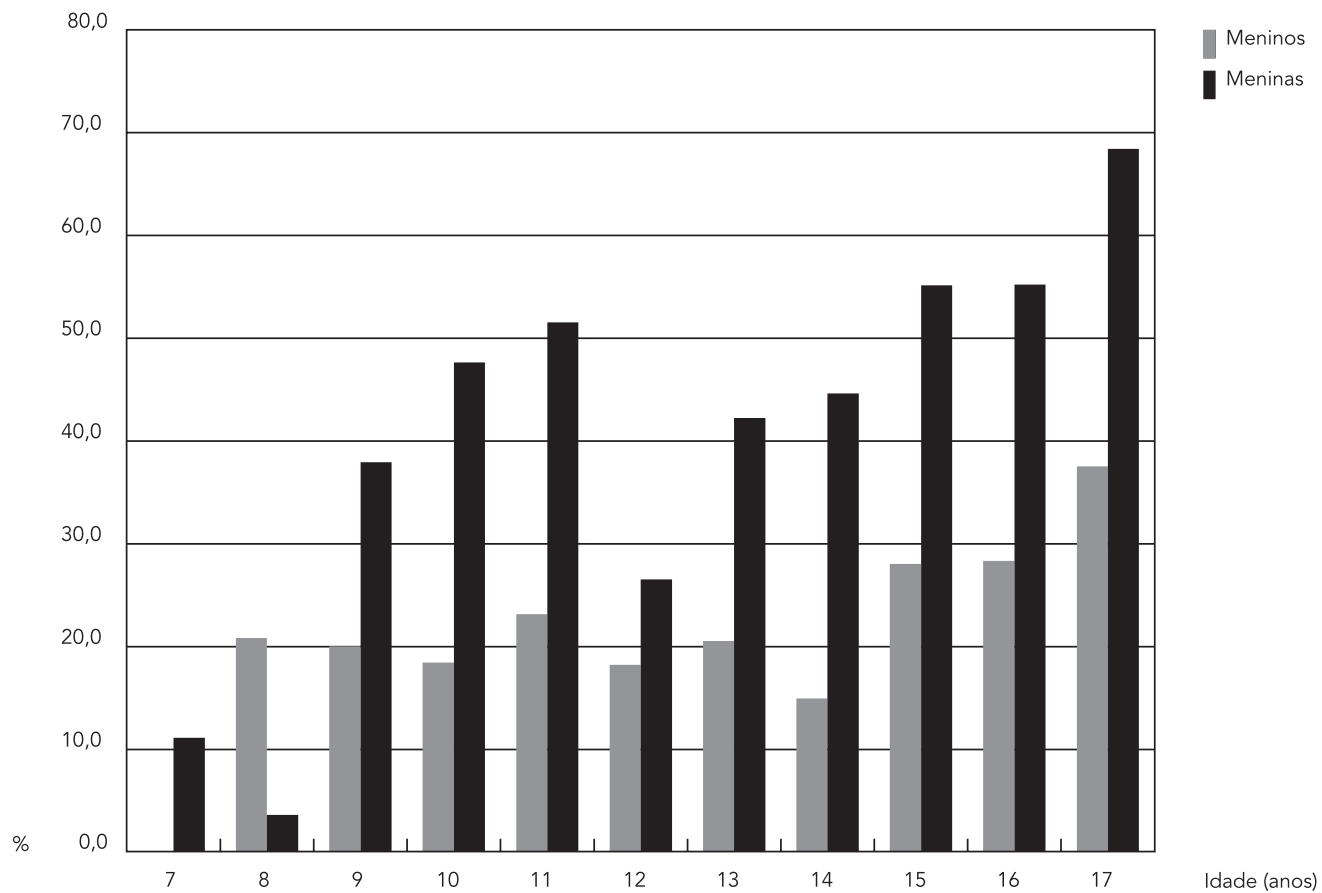


Intensidade da dor lombar, estratificada por sexo.

\begin{tabular}{|c|c|c|c|c|c|c|}
\hline \multirow[t]{2}{*}{ Intensidade } & \multicolumn{2}{|c|}{ Masculino } & \multicolumn{2}{|c|}{ Feminino } & \multicolumn{2}{|c|}{ Total } \\
\hline & $\mathrm{n}$ & $\%$ & $\mathrm{n}$ & $\%$ & $\mathrm{n}$ & $\%$ \\
\hline 1 (dor mínima) & 21 & 25,3 & 18 & 11,2 & 39 & 16,0 \\
\hline 2 & 33 & 39,8 & 64 & 40,0 & 97 & 39,9 \\
\hline 3 & 21 & 25,3 & 50 & 31,3 & 71 & 29,2 \\
\hline 4 & 7 & 8,4 & 20 & 12,5 & 27 & 11,1 \\
\hline 5 (dor máxima) & 1 & 1,2 & 8 & 5,0 & 9 & 3,7 \\
\hline
\end{tabular}

entretanto é possível constatar que, independentemente do estudo, as ocorrências são elevadas e preocupantes. No cenário internacional, elas variam de $23,7 \%$ a $64,8 \%$, considerando algumas investigações dos últimos cinco anos 19,22,25,29,30,31,32.

Em relação à ocorrência de dor lombar ao longo das idades (Figura 1), é possível verificar que, dos 8 aos 13 anos, aproximadamente $20 \%$ dos meninos apresentam dor lombar, e tal percentual aumenta para $28 \%$ aos 15 e 16 anos e para $37 \%$ aos 17 anos. As meninas apresentam ocorrências superiores às dos meninos em todas as idades, exceto aos oito anos. Aos 11, 15, 16 e 17 anos, mais de $50 \%$ das meninas referiram dor lombar, chegando a $68 \%$ aos 17 anos.

Quanto às intensidades de dor lombar, $40 \%$ dos escolares apontaram o nível dois. Quando esses valores são analisados e estratificados por sexo, o percentual de meninas no nível 1 é inferior ao dos meninos, enquanto nos níveis três a cinco é superior, sugerindo que as meninas referiram níveis superiores de intensidades. Os resultados do presente estudo são semelhantes aos encontrados porVidal 25 , que verificou que as maiores intensidades foram referidas nos níveis 2 (37\%) e 3 (33\%), situando-se as indicações na parte inferior da escala.

Encontrou-se associação entre o sexo feminino $(\mathrm{RP}=1,16)$ e a ocorrência de dor lombar, mesmo resultado verificado em outros os estudos: Yao et al. 22 verificaram maior prevalência de dor lombar em meninas do que em meninos chineses, além de diferenças quanto à frequência e natureza da dor; Diepenmaat et al. ${ }^{33}$, que encontraram razão de chance de 1,5 para dor lombar no sexo feminino pelo método Stepwise da Regressão Logística, afirmam que os motivos da relação entre o sexo feminino e a ocorrência de dor lombar permanecem em especulação. Diferenças na percepção dos sintomas entre os sexos e tendência a internalizar problemas psi- cossociais 16,34,35 são possibilidades para essas elevadas prevalências no sexo feminino.

A idade se associou à dor lombar. Exceto aos 8 anos em que não houve significância estatística, dos 9 aos 17 anos as razões de prevalência são superiores quando comparadas aos 7 anos. Os valores mais elevados podem ser observados dos 15 aos 17 anos (Tabela 4). Estudiosos parecem estar de acordo sobre a relação entre o aumento das idades e a ocorrência de dor lombar $12,14,16,34,35,36,37,38$. Tendo em vista esses achados sobre as idades iniciais de ocorrência da dor lombar, sugere-se que haja maior atenção em relação à pesquisa, à prevenção e ao tratamento de dor lombar em crianças em jovens, visto que os estudos concluem que sua ocorrência na adolescência é fator de risco para a dor lombar na vida adulta 7 .

Dentre os aspectos psicossociais, a hiperatividade, tanto na categoria limítrofe $(\mathrm{RP}=1,08)$, quanto na anormal $(\mathrm{RP}=1,09)$ e os sintomas emocionais, na categoria anormal $(R P=1,10)$, associaram-se à dor lombar. Outros aspectos não foram significativos. Nesse sentido, Sjölie 17 encontrou associação entre baixo índice de bem-estar e dor lombar em escolares de 14 a 16 anos. Diepenmaat et al. 33 identificaram que sintomas depressivos e estresse estavam associados à dor no pescoço e nos braços e à dor lombar em adolescentes de 12 a 16 anos. Investigações que utilizaram o mesmo questionário aplicado no presente estudo, também encontraram os fatores psicossociais como associados à dor lombar: maior pontuação total no questionário associou-se à dor nas costas e no pescoço 39; a categoria anormal dos sintomas emocionais, em relação à normal, apresentou razão de chance de 1,65 40; problemas de conduta e sintomas emocionais se associaram à dor lombar em escolares de 11 a 14 anos 20; a variável "problemas de relacionamento com colegas" foi uma das preditoras de dor lombar persistente em estudo de acompanhamento 
Tabela 3

Fatores associados à dor lombar na etapa univariada da regressão de Poisson.

\begin{tabular}{|c|c|c|c|c|c|}
\hline Variável & $\mathrm{n}$ & $\%$ & RP & IC95\% & Valor de $p$ \\
\hline \multicolumn{6}{|l|}{ Sexo } \\
\hline Masculino & 388 & 50,4 & 1,00 & & \\
\hline Feminino & 382 & 49,6 & 1,16 & $1,114-1,227$ & $<0,001$ \\
\hline \multicolumn{6}{|l|}{ Idade (anos) } \\
\hline 7 & 36 & 4,7 & 1,00 & & \\
\hline 8 & 52 & 6,8 & 1,06 & $0,951-1,174$ & 0,305 \\
\hline 9 & 44 & 5,7 & 1,25 & $1,101-1,417$ & 0,001 \\
\hline 10 & 80 & 10,4 & 1,27 & $1,141-1,407$ & $<0,001$ \\
\hline 11 & 72 & 9,4 & 1,29 & $1,157-1,437$ & 0,007 \\
\hline 12 & 78 & 10,1 & 1,15 & $1,041-1,279$ & $<0,001$ \\
\hline 13 & 89 & 11,6 & 1,24 & $1,125-1,379$ & $<0,001$ \\
\hline 14 & 103 & 13,4 & 1,24 & $1,125-1,370$ & $<0,001$ \\
\hline 15 & 99 & 12,9 & 1,34 & $1,214-1,479$ & $<0,001$ \\
\hline 16 & 82 & 10,6 & 1,31 & $1,177-1,449$ & $<0,001$ \\
\hline 17 & 35 & 4,5 & 1,46 & $1,286-1,662$ & $<0,001$ \\
\hline \multicolumn{6}{|c|}{ Prática de exercício } \\
\hline Sim & 246 & 31,9 & 1,00 & & \\
\hline Não & 524 & 68,1 & 1,03 & $0,973-1,082$ & 0,345 \\
\hline \multicolumn{6}{|c|}{ Aspectos psicossociais } \\
\hline \multicolumn{6}{|c|}{ Hiperatividade } \\
\hline Normal & 575 & 74,7 & 1,00 & & \\
\hline Limítrofe & 81 & 10,5 & 1,10 & $1,017-1,196$ & 0,019 \\
\hline Anormal & 114 & 14,8 & 1,13 & $1,058-1,215$ & $<0,001$ \\
\hline \multicolumn{6}{|c|}{ Problemas com colegas } \\
\hline Normal & 573 & 74,5 & 1,00 & & \\
\hline Limítrofe & 149 & 19,4 & 1,05 & $0,981-1,114$ & 0,174 \\
\hline Anormal & 48 & 6,1 & 1,01 & $0,911-1,124$ & 0,826 \\
\hline \multicolumn{6}{|c|}{ Sintomas emocionais } \\
\hline Normal & 603 & 78,3 & 1,00 & & \\
\hline Limítrofe & 88 & 11,4 & 1,03 & $0,947-1,108$ & 0,555 \\
\hline Anormal & 79 & 10,3 & 1,19 & $1,102-1,286$ & $<0,001$ \\
\hline \multicolumn{6}{|c|}{ Problemas de conduta } \\
\hline Normal & 578 & 75,1 & 1,00 & & \\
\hline Limítrofe & 101 & 13,1 & 1,04 & $0,964-1,120$ & 0,314 \\
\hline Anormal & 91 & 11,8 & 1,08 & $1,005-1,173$ & 0,038 \\
\hline \multicolumn{6}{|c|}{ Comportamento pró-social } \\
\hline Normal & 647 & 84 & 1,00 & & \\
\hline Limítrofe & 51 & 6,6 & 0,99 & $0,892-1,090$ & 0,786 \\
\hline Anormal & 72 & 9,4 & 1,04 & $0,952-1,130$ & 0,404 \\
\hline
\end{tabular}

IC95\%: intervalo de 95\% de confiança; RP: razão de prevalência.

de quatro anos ${ }^{9}$ e, também em estudo longitudinal, jovens com problemas de conduta e hiperatividade apresentaram risco aumentado para dor lombar um ano após a avaliação inicial 41.

A prática de exercício para além das aulas de Educação Física não se associou à ocorrência de dor lombar. Skoffer et al. 32 , ao analisarem a prá- tica de diferentes esportes numa amostra de 546 indivíduos de 15 e 16 anos, concluíram que, com exceção da natação, não foram evidenciados resultados que sugiram que maior atividade esportiva previna a dor lombar. No mesmo estudo, a inatividade física se associou ao desfecho. Em investigação com escolares de nove anos de ida- 
Tabela 4

Fatores associados à dor lombar na etapa multivariada da regressão de Poisson.

\begin{tabular}{|c|c|c|}
\hline Variável & RP & IC95\% \\
\hline \multicolumn{3}{|l|}{ Sexo } \\
\hline Masculino & 1,00 & \\
\hline Feminino & 1,16 & $1,106-1,218$ \\
\hline \multicolumn{3}{|l|}{ Idade (anos) } \\
\hline 7 & 1,00 & \\
\hline 8 & 1,04 & $0,931-1,167$ \\
\hline 9 & 1,23 & $1,088-1,394$ \\
\hline 10 & 1,25 & $1,127-1,384$ \\
\hline 11 & 1,29 & $1,159-1,430$ \\
\hline 12 & 1,16 & $1,046-1,288$ \\
\hline 13 & 1,23 & $1,119-1,363$ \\
\hline 14 & 1,21 & $1,096-1,330$ \\
\hline 15 & 1,31 & $1,191-1,449$ \\
\hline 16 & 1,30 & $1,172-1,436$ \\
\hline 17 & 1,42 & $1,256-1,6617$ \\
\hline \multicolumn{3}{|c|}{ Aspectos psicossociais } \\
\hline \multicolumn{3}{|c|}{ Hiperatividade } \\
\hline Normal & 1,00 & \\
\hline Limítrofe & 1,08 & $1,004-1,171$ \\
\hline Anormal & 1,09 & $1,019-1,168$ \\
\hline \multicolumn{3}{|c|}{ Sintomas emocionais } \\
\hline Normal & 1,00 & \\
\hline Limítrofe & 0,96 & $0,885-1,032$ \\
\hline Anormal & 1,10 & $1,023-1,191$ \\
\hline
\end{tabular}

IC95\%: intervalo de 95\% de confiança;

RP: razão de prevalência.

de, reavaliados aos 12 anos, Wedderkopp et al. ${ }^{21}$ concluíram que elevado nível de atividade física na infância, medido por meio de acelerômetros, pareceu proteger contra dor lombar e torácica no início da adolescência. Por outro lado, investigação com 95 meninos de 13 e 14 anos demonstrou que aqueles considerados sedentários apresentaram menor ocorrência de dor lombar do que os regularmente envolvidos em esportes competitivos (basquete, natação e beisebol) 41. Os resultados deste último estudo devem ser interpretados com cautela, tendo em vista que os autores consideraram como sedentários os sujeitos que participavam das aulas de Educação Física escolar, mas que não realizavam esportes fora da escola, comparando-os com um grupo de jovens integrantes de equipes competitivas. No mesmo sentido, o estudo de Vitta et al. ${ }^{24}$ questionou, entre outros aspectos, a escolares de 11 a 14 anos sobre "pratica modalidade esportiva de competição fora da escola?", tendo encontrado razão de chance para dor lombar superior nos que responderam afirmativamente à questão $(\mathrm{OR}=2,58)$. No presente estudo, não classificamos os sujeitos em sedentários ou ativos, tendo em vista que todos participam das aulas de Educação Física escolar. O objetivo foi verificar se havia diferença na razão de prevalência para dor lombar entre o grupo que apenas participava das aulas de Educação Física escolar e aquele que praticava exercício para além dessas aulas. Os diferentes conceitos empregados nesse âmbito dificultam uma comparação mais acurada entre os resultados. Estudos futuros precisam ser desenvolvidos a fim de avaliarem se, em escolares, assim como em estudo com população de adultos e idosos 42, ocorre uma relação entre dor lombar e atividade física em forma de "U", na qual tanto o sedentarismo como níveis demasiadamente elevados de atividade física acarretam no desfecho.

Em síntese, a ocorrência de dor lombar em escolares de 7 a 17 anos de idade foi elevada e as intensidades referidas, preocupantes. As meninas apresentaram razão de prevalência superior à dos meninos. Nas idades de 9 a 17 anos, as razões de prevalência variaram de 1,16 a 1,42, sendo bastante elevadas dos 15 aos 17 anos, quando comparadas aos sete anos. Dentre os aspectos psicossociais investigados, sintomas emocionais e hiperatividade se associaram à presença de dor lombar. Tendo em vista que o presente estudo avaliou crianças e jovens de uma única escola, os achados devem ser extrapolados apenas para escolares com características semelhantes. Uma limitação do estudo, especialmente em relação aos mais jovens, é o fato de o preenchimento do questionário de dor e da prática de exercícios fora da escola depender da recordação dos escolares.

Em investigações futuras, pretendemos verificar a importância de outras variáveis na ocorrência de dor lombar, tendo em vista que o mapeamento de sua ocorrência e etiologia é de fundamental importância para o desenvolvimento de programas eficazes de prevenção primária. 


\section{Resumen}

Los estudios han demostrado que las personas con dolor de espalda en la infancia y la adolescencia también se ven afectadas en la edad adulta, lo que subraya la importancia de que las causas se investiguen desde la edad escolar. Se evaluó la incidencia de dolor de espalda y factores asociados, en 770 escolares de 7 a 17 años en una escuela privada en Porto Alegre, Rio Grande do Sul, Brasil. La lumbalgia se define como un dolor o molestia en la espalda en el último mes y fue evaluada mediante un cuestionario. La aparición de dolor lumbar se produjo en un $31,6 \%$, siendo más frecuente en el sexo femenino $(41,9 \%)$ que en hombres $(21,4 \%)$. Los factores asociados con el dolor de espalda eran: mujeres, edad de 9 a17 años, y aspectos psicosociales como hiperactividad (categorías borderline y anormal) y síntomas emocionales (categoría anormal). El mapeo de la aparición de dolor en la zona baja de la espalda, así como investigar factores asociados, es fundamental para identificar a los niños, niñas y adolescentes en situación de riesgo. con el fin de desarrollar programas eficaces para la prevención primaria.

Dolor de Espalda; Niño; Adolescente; Factores de Riesgo

\section{Colaboradores}

A. T. Lemos contribuiu na concepção do projeto de pesquisa, análise e interpretação dos dados e redação do artigo. F. R. Santos colaborou na análise e interpretação dos dados e revisão crítica do manuscrito. R. B. Moreira participou da análise e interpretação dos dados e revisão crítica do manuscrito. D. T. Machado e F. C. C. Braga contribuíram na análise e interpretação dos dados e na redação do manuscrito. A. C. A. Gaya participou na concepção do projeto de pesquisa e revisão crítica do conteúdo intelectual. Todos os autores participaram da aprovação final da versão a ser publicada.

\section{Agradecimentos}

Ao CNPq pela bolsa de produtividade científica ao autor A. Gaya. À Capes pela bolsa de doutorado à autora A. T. Lemos.

\section{Referências}

1. Burton AK, Balague F, Cardon G, Eriksen HR, Henrotin Y, Lahad A, et al. Chapter 2. European guidelines for prevention in low back pain: November 2004. Eur Spine J 2006; 15 Suppl 2:S136-68.

2. Airaksinen O, Brox JI, Cedraschi C, Hildebrandt J, Klaber-Moffett J, Kovacs F, et al. Chapter 4. European guidelines for the management of chronic nonspecific low back pain. Eur Spine J 2006; 15 Suppl 2:S192-300.

3. Walker B. The prevalence of low back pain: a systematic review of the literature from 1996 to 1998. J Spinal Disord 2000; 13:205-17.

4. Gilgil E, Kaçar C, Butun B, Tuncer T, Urhan S, Yildirim C, et al. Prevalence of low back pain in a developing urban setting. Spine 2005; 30:1093-8.

5. Worku Z. Prevalence of low back pain in Lesotho Mothers. J Manipulative Physiol Ther 2000; 23:147-54.

6. Balagué F, Troussier B, Salminen JJ. Non-specific low back pain in children and adolescents: risk factors. Eur Spine J 1999; 8:429-38.
7. Hestbaek L, Leboeuf-Yde C, Kyvik KO. Is comorbidity in adolescence a predictor for adult low back pain? A prospective study of a young population. BMC Musculoskelet Disord 2006; 7:29.

8. Watson K, Papageorgiou A, Jones G, Taylor S, Symmons DPM, Silman AJ, et al. Low back pain in schoolchildren: occurrence and characteristics. Pain 2002; 97:87-92.

9. Jones GT, MacFarlane GJ. Predicting persistent low back pain in schoolchildren: a prospective cohort study. Arthritis Rheum 2009; 61:1359-66.

10. Andersen LB, Wedderkopp N, Leboeuf-Yde C. Association between back pain and physical fitness in adolescents. Spine (Phila Pa 1976) 2006; 31:1740-4.

11. Balagué F, Mannion AF, Pellisé F, Cedraschi C. Nonspecific low back pain. Lancet 2012; 379:482-91.

12. Bejia I, Abid N, Ben Salem K, Letaief M, Younes M, Touzi M, et al. Low back pain in a cohort of 622 Tunisian schoolchildren and adolescents: an epidemiological study. Eur Spine J 2005; 14:331-6. 
13. Feldman DE, Shrier I, Rossignol M, Abenhaim L. Risk factors for the development of low back pain in adolescence. Am J Epidemiol 2001; 154:30-6.

14. Grimmer K, Williams M. Gender-age environmental associates of adolescent low back pain. Appl Ergon 2000; 31:343-60.

15. Johnson OE, Mbada CE, Agbeja OB, Obembe AO, Awotidebe TO, Okonji AM. Relationship between physical activity and back extensor muscles endurance to the risk of low-back pain in schoolaged adolescents. TAF Preventive Medicine Bulletin 2011; 10:415-20.

16. Shehab DK, Al-Jarallah KF. Nonspecific low back pain in Kuwaiti children and adolescents: associated factors. J Adolescent Health 2005; 36:32-5.

17. Sjolie AN. Psychosocial correlates of low-back pain in adolescents. Eur Spine J 2002; 11:582-8.

18. Sjolie AN. Active or passive journeys and low back pain in adolescents. Eur Spine J 2003; 12:581-8.

19. Turk Z, Vauhnik R, Micetić-Turk D. Prevalence of nonspecific low back pain in schoolchildren in north-eastern Slovenia. Coll Antropol 2011; 35:1031-5.

20. Watson K. Low back pain in schoolchildren: the role of mechanical and psychosocial factors. Arch Dis Child 2003; 88:12-7.

21. Wedderkopp N, Kjaer P, Hestbaek L, Korsholm L. High-level physical activity in childhood seems to protect against low back pain in early adolescence. Spine (Phila Pa 1976) 2009; 9:134-41.

22. Yao W, Mai X, Luo C, Ai F, Chen Q. A cross-sectional survey of nonspecific low back pain among 2083 schoolchildren in China. Spine (Phila Pa 1976) 2011; 36:1885-90.

23. Graup S, Santos SG, Moro ARP. Estudo descritivo de alterações posturais sagitais da coluna lombar em escolares da rede federal de ensino de Florianópolis. Rev Bras Ortop 2010; 45:453-9.

24. De Vitta A, Martinez MG, Piza NT, Simeão SFAP, Ferreira NP. Prevalência e fatores associados à dor lombar em escolares. Cad Saúde Pública 2011; 27:1520-8.

25. Vidal AR. Dor lombar inespecífica em alunos adolescentes em função do género, idade e nível de actividade física [Dissertação de Mestrado]. Porto: Universidade do Porto; 2009.

26. Jones GT, Watson KD, Silman AJ, Symmons DPM, MacFarlane GJ. Predictors of low back pain in British schoolchildren: a population-based prospective cohort study. Pediatrics 2003; 111:822.

27. Goodman R. The Strengths and Difficulties Questionnaire. A research note. J Child Psychol Psychiatriy 1997; 38:581-6.

28. Strengths and Difficulties Questionnaire (SDQ). http://www.sdqinfo.com/py/sdqinfo/b3.py?la nguage $=$ Portugueseqz (Brazil) (acessado em 05/ Mai/2012).

29. Auvinen J, Tammelin T, Taimela S, Zitting P, Karppinen J. Associations of physical activity and inactivity with low back pain in adolescents. Scand J Med Sci Sports 2008; 18:188-94.
30. Balagué F, Ferrer M, Rajmil L, Pont Acuña A, Pellisé F, Cedraschi C. Assessing the association between low back pain, quality of life, and life events as reported by schoolchildren in a population-based study. Eur J Pediatr 2012; 171:507-14.

31. Sato T, Ito T, Hirano T, Morita O, Kikuchi R, Endo N, et al. Low back pain in childhood and adolescence: a cross-sectional study in Niigata City. Eur Spine J 2008; $17: 1441-7$.

32. Skoffer B, Foldspang A. Physical activity and lowback pain in schoolchildren. Eur Spine J 2008; 17:373-9.

33. Diepenmaat A, Van der Wal M, de Vet H, Hirasing R. Neck/shoulder, low back, and arm pain in relation to computer use, physical activity, stress, and depression among Dutch adolescents. Pediatrics 2006; 117:412-6.

34. Brack C, Brack G, Orr D. Dimensions underlying problem behaviors emotions and related psychosocial factors in early and middle adolescents. J Early Adolesc 1994; 14:345-70.

35. McGrath P. Psychological aspects of pain perception. Arch Oral Biol 1994; 39 Suppl:55S-62S.

36. Hestbaek L, Leboeuf-Yde C, Kyvik KO, Vach W, Russell MB, Skadhauge L, et al. Comorbidity with low back pain: a cross-sectional population-based survey of 12- to 22-year-olds. Spine (Phila Pa 1976) 2004; 29:1483-91.

37. El-Metwally A, Salminen JJ, Auvinen A, Kautiainen H, Mikkelsson M. Prognosis of non-specific musculoskeletal pain in preadolescents: a prospective 4-year follow-up study till adolescence. Pain 2004; 110:550-9.

38. Sundblad GMB, Saartok T, Engström L-MT. Prevalence and co-occurrence of self-rated pain and perceived health in school-children: age and gender differences. Eur J Pain 2007; 11:171-80.

39. Adamson G, Murphy S, Shevlin M, Buckle P, Stubbs D. Profiling schoolchildren in pain and associated demographic and behavioural factors: a latent class approach. Pain 2007; 129:295-303.

40. Murphy S, Buckle P, Stubbs D. A cross-sectional study of self-reported back and neck pain among English schoolchildren and associated physical and psychological risk factors. Appl Ergon 2007; 38:797-804.

41. Balagué F, Bibbo E, Mélot C, Szpalski M, Gunzburg $\mathrm{R}$, Keller TS. The association between isoinertial trunk muscle performance and low back pain in male adolescents. Eur Spine J 2010; 19:624-32.

42. Heneweer H, Vanhees L, Picavet HSJ. Physical activity and low back pain: a U-shaped relation? Pain 2009; 143:21-5

Recebido em 16/Fev/2013

Versão final reapresentada em 26/Mai/2013

Aprovado em 05/Jun/2013 